\title{
Devaney Chaos Induced by Turbulent and Erratic Functions
}

\author{
Vincent. N. $S^{1}$, Vinod Kumar ${ }^{2}$ \\ ${ }^{1}$ (Department of Mathematics, Christ University, India) \\ ${ }^{2}$ (Department of Mathematics, Rajagiri School of Engineering \& Technology, India)
}

\begin{abstract}
Let I be a compact interval and fbe a continuous function defined from I to I. We study the relationship between tubulent function, erratic function and Devaney Chaos.
\end{abstract}

Keywords: Devaney Chaos, Erratic Function, Turbulent Function

\section{Introduction}

After the introduction of first mathematical definition of chaos in 1975 by Li and Yorke [3], several other definitions in various contexts were introduced. The most popular among all such definitions is the one given by Robert. L. Devaney [4]. In Devaney's definition of chaos these are three important factors, namely, Transitivity, Dense periodicity and Sensitivity. In this manuscript we try to prove the relationship between turbulent function, erratic function and Devaney chaos. Firstly we study the relation between turbulence and chaotic function and in the second part, the interconnection between the concepts given in the keywords.

\subsection{Definition: Devaney Chaos}

\section{Preliminaries}

Let $V$ be a set. $f: V \rightarrow V$ is said to be chaotic on $V$ if

- $f$ has sensitive dependence on initial conditions

- $f$ is topologically transitive

- periodic points are dense in $V$

\subsection{Definition: Turbulent Function}

Let $f: X \rightarrow X$ be a continuous function. We say that $f$ is a turbulent function if there exist compact subintervals $J, K$ with at most one common point such that $J \cup K \subseteq f(J) \cap f(K)$.

2.3. Definition: $f: J \rightarrow J$ is said to be topologically transitive if for any pair of open sets $U, V \subset J$ there exists $k>0$ such that $f^{k}(U) \cap V \neq \varnothing$

2.4. Definition: $f: J \rightarrow J$ has sensitive dependence on initial conditions if there exists $\delta>0$ such that, for any $x \in J$ and any neighbourhood $N$ of $x$, there exists $y \in N$ and $n \geq 0$ such that $\left|f^{n}(x)-f^{n}(y)\right|>\delta$.

\subsection{Definition: Erratic Function}

Suppose that $\mathrm{X}$ is a compact convex subset of a linear space and let $f: X \rightarrow X$ be a continuous function. We say that $\mathrm{f}$ is an erratic function if there exists a nonempty convex compact subset $\mathrm{A}$ of $\mathrm{X}$ such that

a) $\mathrm{A} \cap \mathrm{f}(\mathrm{A})=\varnothing$

b) $\mathrm{A} \cup \mathrm{f}(\mathrm{A}) \subseteq \mathrm{f}^{2}(\mathrm{~A})$

\subsection{Definition: B-C Chaotic}

Let $f: X \rightarrow X$ be a continuous function. We say that $f$ is chaotic in the sense of Block and Coppel (B-C-chaotic) if one of its iterates is turbulent, i.e., there exist $\mathrm{n} \geq 1$ and two disjoint nonempty compact subsets $\mathrm{J}, \mathrm{K}$ of $\mathrm{X}$ such that $J \cup K \subseteq f^{n}(J) \cap f^{n}(K)$

2.7. Result [1]

Let $f: X \rightarrow X$ be a continuous function. If $f$ is an erratic function, then $f$ is a $\mathrm{B}-\mathrm{C}$ Chaotic function.

2.8. Result [2]

A continuous map $f: I \rightarrow I$ on an interval is $\mathrm{D}$ - chaotic if and only if it is $\mathrm{B}-\mathrm{C}$ chaotic.

2.9. Result [5] (Chapter II, p.26)

If $\mathrm{f}$ is turbulent, then there exist points $a, b, c \in I$ such that $f(b)=f(a)=a, f(c)=b$ and either $a<c<$ $b, f(x)>$ a for $a<x<b$

$$
\begin{gathered}
x<f(x)<b \text { for } a<x<c \\
\text { or } b<c<a, f(x)<a \text { for } b<x<a \\
b<f(x)<x \text { for } c<x<a
\end{gathered}
$$


2.10. Result [5] (Chapter IV, p.75)

If $J$ is a subinterval of $I$ which contains no periodic point of $f$ then, for any $x \in I$, the points of the trajectory $\gamma(x)$ which lie $\mathbf{J}$ form a strictly monotonic.

\subsection{Theorem}

\section{Main Results}

If $f: I \rightarrow I$ be a continuous map and $f$ is turbulent, then $f$ is Devaney Chaotic.

Proof: If $f$ is a turbulent function, so it must satisfy the necessary and sufficient condition for the turbulent function which is given in Lemma 2.9 .

If $\mathrm{f}$ is turbulent, then there exist points $a, b, c \in I$ such that $f(b)=f(a)=a, f(c)=b$ and either $a<c<$ $b, f(x)>a$ for $a<x<b$

$$
\begin{gathered}
x<f(x)<b \text { for } a<x<c \\
\text { or } b<c<a, f(x)<a \text { for } b<x<a \\
b<f(x)<x \text { for } c<x<a
\end{gathered}
$$

Suppose the pair of open sets be $(a, c)$ and $(c, b)$, So from the necessary condition for turbulence we have $a<c<b$,

$$
\begin{gathered}
f(x)>\text { a for } a<x<b \\
x<f(x)<b \text { for } a<x<c
\end{gathered}
$$

So after successive iterations the elements of $(a, c)$ will enter to the second open set $(c, b)$

So we can conclude that $f$ is Turbulent $\Rightarrow f$ is Topologically Transitive.

We know that If $\mathrm{f}$ is turbulent, then there exist points $a, b, c \in I$ such that $f(b)=f(a)=a, f(c)=b$. So there exist compact subintervals $J, K$ with at most one common point. Let $J=[\alpha, \beta]$ and $K=[\gamma, \delta]$, where $\beta=\gamma$. Then we assume that $f(\beta) \neq \beta$. Now $[\alpha, \delta]$ be the neighbourhood of $\beta$ with $>0$. Since the function is turbulent, we have $|f(\beta)-f(\alpha)|=\delta_{1}>\delta$. Then $f$ has sensitive dependence on initial conditions.

In order to prove the dense set of periodic points, we have to show that each open interval contains a periodic point. Let $U$ be an open set in $I$. Since $\mathrm{f}$ is turbulent, then it implies that $J \cup K \subseteq f(J) \cap f(K)$. Given any $n \in N, \exists x_{n}$ such that $f^{n}\left(x_{n}\right)=x_{n}$.

Suppose there are no periodic points in $U$.

$$
\begin{aligned}
& \forall x \in U, f^{n}(x) \neq x, \quad \forall n \\
& \Rightarrow \nexists y, z \in J, K \text { such that } f^{n}(y)=f^{m}(z)=x \quad \forall n, m \\
& \Rightarrow f(x)=f^{n+1}(y)=f^{m+1}(z) \\
& \Rightarrow f^{k}(x)=f^{n+k}(y)=f^{m+k}(z) \\
& \Rightarrow O_{f}(x)=\left\{\mathrm{x}, f(x), f^{2}(x), \ldots \ldots, f^{k}(x)\right\} \cap \mathrm{U}=\emptyset
\end{aligned}
$$

So we have arrived with a contradiction that $f$ is not transitive. But if $f$ is turbulent then $f$ is transitive. So $U$ has periodic points and therefore the set of periodic points of $f$ are dense in I.Then $f$ is Devaney Chaotic.

Theorem 3.2.

If $f$ is a continuous map from an interval to itself and $f$ is an erratic function implies $f^{n}$ is turbulent, $n \geq 2$.

Proof: Suppose that $\mathrm{f}$ is an erratic function and let us take $J=A$ and $K=f(A)$.

So $J \cup K=A \cup f(A) \subseteq f^{2}(A)=f(f(A))=f(K)$

We know that $f(K) \subseteq f(J \cup K) \subseteq f\left(f^{2}(J)=f^{3}(J)\right.$

Also we know that $f(J \cup K) \subseteq f\left(f^{2}(K)=f^{3}(K)\right.$

This inclusion continuous for $n \geq 2$, where $n$ is a non negative integer

Therefore $f^{n}$ is turbulent.

Theorem 3.3.

If $f$ is a continuous map from an interval to itself and $f$ is erratic implies $f$ is topologically transitive and sensitive dependence on initial conditions.

Proof: Since $A \cup f(A)=\emptyset$ and $f(A) \subseteq f^{2}(A)$, if we take any two open sets we can attain the condition, $f^{k}(U) \cap V \neq \varnothing$. So the function is topologically transitive. Since $A \subseteq f^{2}(A)$, if we have a $\delta$ - neighbourhood at $x \in A$ and $y \in N_{\delta}(x)$, after iteration we will have $|f(x)-f(y)|=\delta_{1}>\delta$. The function is s. d. i. $c$ Theorem 3.4.

If $f$ is a continuous map from an interval to itself and $f$ is erratic implies $f$ is a Devaney Chaotic function. Proof: Using the results 2.7. \& 2.8., we can prove the theorem. Hence the proof 


\section{References}

\section{Journal Papers:}

[1]. H. Roman Flores, Y. Chalco - Cano and G. Nunes Silva, Chaos induced by Turbulent and Erratic functions, IFSA - EUSFLAT. 2009, $231-233$

[2]. B. Aulbach and B. Kieninger, On Three Definitions of Chaos, Nonlinear Dynamics and Systems Theory, 1 (1), 2001, 23 - 37.

[3]. T. Y. Li and J. A. Yorke, Period three implies Chaos, Amer. Math. Mon. 82, 1975, 985 - 992.

Books:

[4]. R. L. Devaney, An introduction to Chaotic Dynamical systems, Addison - Wesley, Redwood city, CA $2^{\text {nd }}$ edition (1989).

[5]. L.S. Block and W.A. Coppel, Dynamics in One Dimension, Lecture Notes in Mathematics no. 1513, Springer - Verlag 1992. 